\title{
Percepções de Adolescentes Escolares sobre Saúde e Meio Ambiente para Práticas Sustentáveis e Promotoras de Saúde
}

\section{Sabrina Alaide Amorim¹ Alves, Italla Maria Pinheiro Bezerra², Edilma Gomes Rocha Cavalcante ${ }^{3}$, Greyce Alencar Albuquerque ${ }^{3}$, Maria do Socorro Vieira Lopes ${ }^{3}$}

\author{
${ }^{1}$ Mestrado Acadêmico em Enfermagem da Universidade Regional do Cariri, Ceará, Brasil | \\ sabrina1995amorim@gmail.com | https://orcid.org/0000-0001-5831-4668 \\ ${ }^{2}$ Escola Superior de Ciências da Santa Casa de Misericórdia de Vitória (EMESCAM). Vitória \\ Espirito Santo,Brasil | itallamaria@hotmail.com | https://orcid.org/0000-0002-8604-587X \\ 3Docente Mestrado Acadêmico em Enfermagem da Universidade Regional do Cariri, \\ Ceará, Brasil | edilma.rocha@yahoo.com.br; geycyenf.ga@gmail.com; \\ socorrovieira@hotmail.com | https://orcid.org/0000-0002-6861-2383; \\ https://orcid.org/0000-0002-8726-0619; https://orcid.org/0000-0003-1335-5487
}

Resumo: Conhecer a percepção de adolescentes sobre a relação saúde e meio ambiente para práticas sustentávies e promotoras de saúde. Método: Estudo qualitativo, conduzido de acordo com COREQ. Os dados foram coletados no mês de maio de 2020, por meio de uma entrevista semiestrutura, com a participação de 13 adolescentes de uma escola de ensino Fundamental II inserida no território do Geopark Araripe, de um município localizado na Microrregião do Cariri, Nordeste, Ceará, Brasil. Os dados foram analisados segundo a técnica análise de conteúdo. Resultados: Os depoimentos revelam que para os adolescentes a relação saúde e meio ambiente é permeada por temáticas como poluição, desmatamento e mudanças climáticas, implicando o distanciamento quanto à conceituação ampliada de saúde. Conclusão: $O$ estudo aponta que a relação saúde e meio ambiente dever ser pautada por temas transversais e pluraristas, de modo a reconhecer o ambiente como espaço vivo.

Palavras-chave: Adolescente; Tecnologia em Saúde; Meio Ambiente; Saúde.

Perceptions of School Teenagers on Health and the Environment for Sustainable Practices and Health Promoters

Abstract: To know the adolescents' perception about the health and environment relationship for sustainable and health-promoting practices. Methods: Qualitative study, conducted according to COREQ. Data were collected in May 2020, through a semi-structured interview, with the participation of 13 adolescents from an elementary school in a municipality located in the Microregion of Cariri, Northeast, Ceará, Brazil. The data were analyzed according to the content analysis technique. Results: The testimonies reveal that for adolescents, the relationship between health and the environment is permeated by themes such as pollution, deforestation and climate change, implying a detachment regarding the expanded concept of health. Conclusion: The study points out that the relationship between health and the environment must be guided by transversal and pluralistic themes, in order to recognize the environment as a living space.

Keywords: Adolescent; Health Technology; Environment; Health.

\section{Introdução}

Depreende-se a adolescência como um período marcado por construção sócio-histórica, cujas manifestações estão fortemente influenciadas pelos fatores socioeconômicos, políticos e culturais do meio onde o adolescente vive (Brasil, 2013; Machado, 2015). Nessa perspectiva, destaca a necessidade de elaborações e construções de políticas públicas de promoção da saúde aos adolescentes direcionadas aos alcances das metas do milênio, já que essa população é considerada uma janela de oportunidades para o ser saudável e produtivo na idade adulta (Usa, 2011; Farre, 2018).

Desta forma, os Objetivos do Desenvolvimento Sustentável (ODS), procura tornar realidade o princípio da indivisibilidade dos direitos humanos quando exigem a realização prática dos direitos econômicos, sociais e culturais, mesmo em realidades tão distintas de países considerados desenvolvidos e em desenvolvimento (Silva, 2018). 
Nessa perspectiva, a saúde torna-se um indicador do progresso de nações no alcance ao desenvolvimento sustentável (Fairall, 2017), contudo promover saúde, em todas as idades, inserem benefícios que se estendem através das gerações. Desse modo, a inserção da saúde como um dos ODS traz o reconhecimento de ser um elemento-chave ao desenvolvimento humano, com vastos e multidimensionais determinantes sociais a grupo em condições vulneráveis a saúde (Djonú, 2018).

O objeto saúde deve incidir diretamente na identificação dos determinantes sociais da saúde, de modo a favorecer a criação de ambientes saúdávies com o objetivo de melhorar a qualidade de vida por meio de mudanças no estilo de vida (Porto, 2006; Bezerra, 2017; Schaefer, 2018; Guha, 2019).

A identificação dos determinantes sociais da saúde permite examinar a relação de quais condicionantes interfere ao alcance das metas do ODS saúde aos adolescentes (Buss, 2016). Apontando a necessidade de práticas em saúde, que possa permitir ao público adolescente uma maior compreensão quanto à relação saúde e meio ambiente. Desta forma, o estudo objetiva conhecer a percepção de adolescentes sobre a relação saúde e ambiente para práticas sustentávies e promotoras de saúde.

\section{Método}

Estudo descritivo, desenvolvido a partir de abordagem qualitativa, conduzido de acordo com a guideline internacional para pesquisas qualitativas COREQ (Critérios consolidados para relato de pesquisa qualitativa), obedecendo aos 32 itens estabelecidos pelo checklist) (Tong, 2007).

O estudo foi desenvolvido em uma escola de ensino fundamental II inserida no território do Geopark Araripe, situada na Microrregião do Cariri, Mesorregião Sul do estado do Ceará, Nordeste, Brasil. Os participantes da pesquisa foram adolescentes, selecionados por conveniência, partindo do pressuposto que os mesmos já possuem algum conhecimento acerca da temática abordada e por amostragem bola de neve. Foram critérios de inclusão ser adolescente entre 11 e 19 anos conforme Organização Mundial de Saúde (OMS); está regularmente matriculado. Como critério de exclusão: após três tentativas de contato com o adolescente sem retorno esse era excluído da pesquisa. Ao final, participaram do estudo 13 adolescentes.

A produção dos dados ocorreu no mês de maio de 2020, a partir de entrevistas semiestruturadas, audiogravadas, realizadas mediante ligações telefônicas, em virtude da pandemia da COVID-19 vivenciada no mundo, no sentido de garantir medidas preventivas de isolamento e seguindo recomendações da Organização Mundial de Saúde (OMS), quanto ao distanciamento social.

Desta forma, a pesquisadora entrava em contato com o adolescente por meio de contato repassado pela coordenação da escola, o primeiro contato era realizado com o responsável para explanação da pesquisa e solicitação de autorização. Pois concedimento do responsável e adolescente era agendado a realização da entrevista, conforme a disponibilidade do adolescente.

Àqueles que aceitaram participar da pesquisa, foram dadas as informações sobre a pesquisa, bem como foi solicitada a sua concordância em participar do estudo e do seu responsável, através da assinatura do Termo de Consentimento Livre e Esclarecido TCLE e Termo de Assentimento Livre Esclarecido (TELC). Os termos foram enviados como questionário do Google Forms como mensagem para o aplicativo WhatsApp.

Para coleta dos dados os horários das entrevistas foram variados, manhã e tarde, de acordo com a disponibilidade dos adolescentes. As ligações telefônicas foram gravadas, com duração média de 15 minutos. Acrescente-se que as entrevistas foram transcritas na íntegra, imediatamente após a realização das mesmas, a fim de não perder informações relevantes para análise dos dados. 
A análise dos dados ocorreu concomitante à coleta, adotando a técnica de análise de conteúdo proposta por Bardin (2010), e, conforme preconizado, executando as três fases: a pré-análise, em que se buscou uma primeira impressão dos dados coletados, que por sua vez subdivide-se em três etapas, a saber: leitura flutuante, a escolha dos documentos que foram organizados para a construção dos corpus da pesquisa que se constituiu de 13 entrevistas com adolescente, hipóteses, os objetivos e a elaboração de indicadores; a fase exploratória, em que o material foi codificado em categorias por meio de recortes dos registros; e a terceira e última fase, ocorreu o tratamento dos resultado obtidos e interpretação.

O estudo foi aprovado pelo Comitê de Ética em Pesquisa da Universidade Regional do Cariri (URCA), sob Parecer n. 3. 839. 083, obedecendo aos princípios da Resolução n. 466/12 do Conselho Nacional de Saúde.

\section{Resultados}

Fizeram parte deste estudo 13 adolescentes, sendo 11 do sexo feminino e dois do sexo masculino, na faixa etária de 12 a 14 anos. Quanto à escolaridade dois cursavam o $7^{\circ}$ ano, dois o $8^{\circ}$ ano e 09 adolescentes cursavam o 9 ano do ensino Fundamental II. No que concerne ao número de pessoas que compõem o núcleo familiar é composta de três a seis pessoas.

Referente à análise das percepções sobre a temática, emergiram as categorias: relação saúde e meio ambiente, temáticas, Saúde Ambiental no contexto escolar. A figura 1 apresenta as evidências do estudo e categorias.

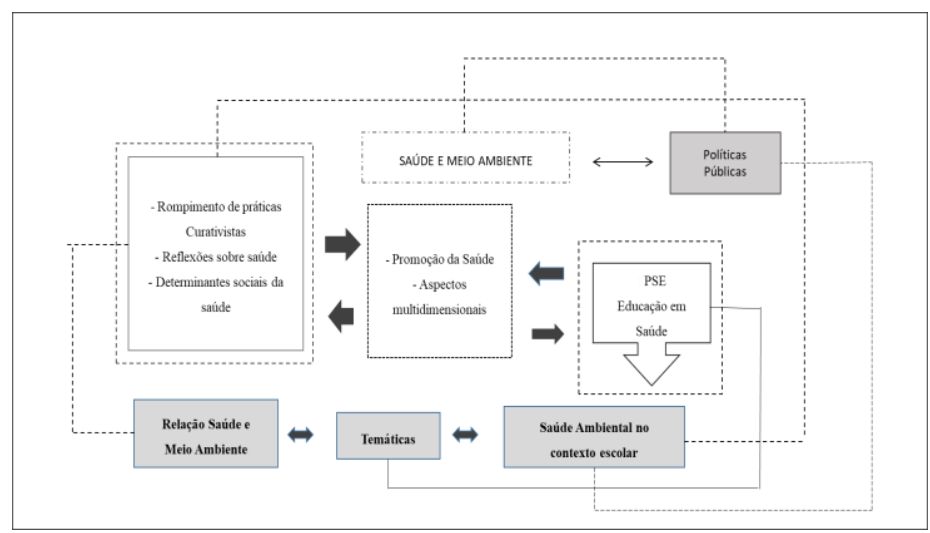

Fig. 1. Evidências do estudo e categorias temáticas segundo técnica de Bardin. Crato, Ceará, Brasil, 2020.

\subsection{Relação Saúde e Meio Ambiente}

Para os adolescentes, a relação saúde e meio ambiente é concebida por uma, percepção focalizada apenas na doença, sem problematizar os determinantes e condicionantes que interferem no processo saúde-doença.

"Saúde é uma coisa que você tem, tipo você está doente, meio ambiente é onde nós convive, tem árvore, tem casa, água, animais". (ADOL 4).

"Saúde é tipo você ter uma saúde estável, você ter uma saúde boa, você cuidar da sua saúde, não comer alimentos que faz mal. Meio ambiente é você conservar as plantas, você conservar as coisas, não jogar lixo no mar, não jogas lixos nas plantas". (ADOL10). 


\subsection{Temáticas}

Observa-se que as temáticas focalizam aspectos limitados quanto a relação saúde e meio ambiente, o que distancia quanto a compreensão do conceito ampliado de saúde. Implicando para práticas de saúde voltadas para o assistencialismo, contrapondo-se aos pressupostos da promoção da saúde.

"[...] poluição" (ADOL 1).

'’...]desmatamento, urbanização e o ambiente, extinção de espécies, mudanças climáticas" (ADOL 8)

"Que você não precisa poluir os rios e tudo" (ADOL 12).

\subsection{Saúde Ambiental no Contexto Escolar}

Os adolescentes reconhecem o ambiente escolar como espaço privilegiado para ações educativas para transmissão de informações e promoção da saúde com temáticas sobre saúde e meio ambiente.

"A escola é um local onde os professores ensinam e onde a gente aprende. E então eu acho que através dos professores ensinando o aluno ele pode aprender a ter uma boa convivência com o meio ambiente e cuidar do lugar onde ele mora e vive, né?" (ADOL 2).

"[...] escola é o inicio da aprendizagem da pessoa. Então quando os professores passam as informações para a pessoa você pode levar tipo, informação para você e para outras pessoas. Para dizer para elas que é para cuidar de sua saúde e não contaminar o meio ambiente" (ADOL 10).

\section{Discussão}

No presente estudo, identificou-se a percepção de adolescentes sobre a relação saúde e meio ambiente para práticas sustentáveis e promotoras de saúde, contudo observou que para os adolescentes o conceito de saúde está relacionado à ausência de doença. Nessa direção, emerge a necessidade dessa população compreender que o conceito de saúde envolve fenômenos sociais, políticos, econômicos e ambientais (Anhas, 2017).

Destaca a necessidade de um olhar além do modelo tradicional de saúde, referindo-se saúde como ausência de doença, posto que se faz necessário refletir sobre os mecanismos que concerne o objeto saúde. No entanto, entende-se a necessidade de discussões acerca da relação saúde e meio ambiente como um desafio no âmbito da saúde coletiva, considerando as vulnerabilidades às doenças, econômicas, socias e exposição ambiental e seus efeitos sobre a saúde da população adolescente (Rangel, 2015).

É preciso compreender que a relação saúde e meio ambiente transcende a perspectiva de uma assistência biologicista, emergindo a necessidade de reproduzir uma visão que busque incorporar conhecimentos referentes aos aspectos relacionados ao processo saúde-doença. Estudo realizado com adolescentes escolares, infere que a visão limitada dessa população quanto a concepção sobre saúde, os torna passivos no que conercer ao seu processo saúde-doença (Oliveira, 2018).

Nessa perspectiva, o ODS saúde vem corroborar com a necessidade de compreensão do conceito ampliado de saúde, concentrando-se em uma abordagem em saúde horizontalizada e sistemática, buscando identificar os condicionantes e determinantes que interfere na saúde (Lee, 2019). 
Os achados corroboram com estudo que reconhece que o conceito de saúde ultrapassa a concepção limitada de doença, flagrando-se a necessidade de uma compreensão embasada em aspectos multidimensionais quanto ao contexto saúde e seus diversos fatores que influenciam na consolidação do processo saúde-doença. Assim, deve-se refletir de forma crítica sobre a influência do meio ambiente como ponto importante para o entendimento desses aspectos que envolve a saúde (Alves, 2018).

A ocorrência quanto a acepção da relação saúde e meio ambiente expressas nas falas dos adolescentes, aponta para conceitos pré-definidos voltados para um caráter biologicista e uma visão restrita da ecologia. Nessa face, o aprofundamento dos debates acerca dessa inter-relação, requer a compreensão que o ambiente perpassa por questões sociais e culturais, em que o indivíduo passa a contemplar uma relação de interdependência com o ambiente (Busato, 2015).

Assim, o ambiente deve ser compreendido como um espaço vivo, consequente de transformações que interferem na condição de saúde da população, requerendo conceito pluralista quanto a sua análise e interpretação. A incorporação e implementação das metas do ODS 3 no alcance e construção da saúde deve incidir na interface entre saúde e ambiente, com vista ao alcance de uma vida saudável pela população, de modo a produzir mudanças sociais e qualidade de vida (Kruk, 2018; Machado, 2017).

Para tanto, a predominância desse olhar curativista implica a necessidade de rompimento com o modelo de saúde vigente, partindo do pressuposto que os determinantes sociais da saúde refletem de forma concreta na interferência dos fenômenos da saúde.

Nesse contexto, é preciso considerar que a compreensão da relação saúde e meio ambiente envolve diferentes e diversas temáticas, que permite embasamento quanto a conceituação ampliada de saúde. Nessa perspectiva, a abordagem dessa relação saúde e meio ambiente deve ser concedida por temáticas que potencialize e busque a ampliação quanto aos condicionantes e determinantes que interfere na saúde do público adolescente (Dias, 2018).

Aponta-se a necessidade de uma abordagem pluralista quanto a relação saúde e meio ambiente, propondo conceber ao adolescente que compreenda de modo amplo e abrangente, temáticas que envolvem essa relação. Nesse sentido, é preciso considerar que saúde e meio ambiente busca articular-se com temas que não se restringe apenas a poluição, desmatamento, mudanças climáticas, mas que possa identificar os processos de produção de saúde (Pereira, 2021).

Os resultados encontrados corroboram com estudo realizado em um municipio do sudoeste da Bahia que reconhece à importancia de temáticas que proporcionam o desenvolvimento de um cuidado interdisciplinar, de modo a identificar necessidades que vão além de uma concepção biomédica, superando práticas assistenciais hegemônicas em saúde, o que muitas vezes são evidenciadas em ações e políticas aos adolescentes (Fernandes, 2020).

A abordagem de temáticas que englobam a relação saúde e meio ambiente deve incidir diretamente a mudanças de atitudes e comportamentos individuais e coletivos, com o reconhecimento de que esses construtos são onipresentes e, por isso, podem repercutir diretamente na saúde. Deste modo, defende-se à importancia do desenvolvimento de práticas educativas como uma ferramenta para se alcançar a promoção da saúde e como uma mudança de paradigma na práxis das práticas de saúde (Jing, 2016; Santos, 2019).

A educação em saúde baseia-se nos pressupostos da promoção da saúde, que busca o desenvolvimento por meio de técnicas e métodos que favoreçam a transformação e emancipação dos sujeitos. De modo, a proporcionar uma reflexão sobre comportamentos saudáveis e sobre as bases socias de sua vida, em uma perspectiva de desenvolvimento de práticas em saúde horizontalizadas (Buss, 2000; Chiesa, 2007).

Nesse enfoque, o desenvolvimento de prática em saúde para o adolescente deve apoiarse em uma pedagogia libertadora, como uma ferramenta de transformação social, uma vez que tenta romper com práticas curativistas. 
Propondo transformar o modelo tradicional de educação em saúde, predominantemente centradas na prevenção de doenças, para uma prática voltada para emancipação e empoderamento dos sujeitos, favorecendo o diálogo (Freire, 2011; Araujo, 2018; Alves, 2019).

Nesse sentido, reconhece o ambiente escolar como cenário para a implementação e desenvolvimento de prática de educação em saúde que possibilite ao adolescente o reconhecimento acerca da relação saúde e meio ambiente como uma relação interdependente e necessária a vida.

Desta forma, a escola torna-se um espaço privilegiado para ações educativas para troca de informação para promoção da saúde do adolescente. Destacando a importancia de práticas educacionais que favoreçam discussões e reflexões acerca de temas voltados para a relação saúde e meio ambiente como forma de empoderar esse público quanto a necessidade de mudanças de atitudes, e do desenvolvimento de práticas em saúde voltadas para ações promotoras de saúde (Monteiro, 2017; Pereira, 2021).

Nesse contexto, destaca o Programa Saúde nas Escolas (PSE), política que tem como objetivo a implementação e desenvolvimento de ações articuladas de saúde e educação, que busque contribuir para inserção de práticas voltadas para promoção da saúde. Acrescenta-se a intersetorialidade como pilar do PSE, como o fortalecimento e enfrentamento das vulnerabilidades em saúde que comprometem o desenvolvimento de ações voltadas para a população adolescente (Brasil, 2016; Baggio, 2018).

Entretanto, revela-se lacunas quanto às ações de promoção da saúde e prevenção de doenças no contexto do PSE, cujas práticas de saúde ainda são fragmentadas, a saber: saúde reprodutiva, vacinação, nutrição, entre outros. Apontando, para uma perspectiva que requer uma investigação quantos as reais necessidades de saúde dessa população. Embora as ações de promoção da saúde estejam explícitas nas práticas de saúde do PSE, emerge a necessidade pela diversidade de temáticas como saúde ambiental, permitindo ao adolescente uma compreensão totalizadora quanto aos determinantes sociais da saúde, de modo que possam ser capazes de interferir quanto ao processo saúde-doença (Machado, 2015; Lopes, 2018).

Estudo desenvolvido revela que face às dificuldades de implementação e desenvolvimento de práticas voltadas para promoção da saúde ao adolescente no contexto do PSE, está a não intersetorialidade de setores para o desenvolvimento de ações relativas à saúde do adolescente. $\mathrm{O}$ desenvolvimento de práticas promotoras de saúde deve compreender a pluralidade e complexidade dos elementos que se relacionam ao processo saúde-doença (Brasil, 2017).

A atenção à saúde dispensada ao público adolescente expressa a necessidade de rompimento de uma visão reducionista e assistencialista, de modo, a considerar o adolescente não apenas como um gerador de problemas, mas com potencialidades para a construção de um sujeito com capacidade de transformação quanto a sua condição de saúde (Shacfer, 2018).

Nota-se nos resultados a necessidades de práticas de educação em saúde que abordem conteúdos que transversalizam a interrelação entre saúde e meio ambiente. Em uma perspectiva de 0 adolescente reconhecer 0 objeto saúde de forma polissêmica e multidimensional sobre as intervenções que o meio ambiente ocasiona no processo saúde-doença (Ventura, 2020).

Faz-se necessário avançar quanto à concepção da relação saúde e meio ambiente, como forma de gerar reflexões capazes de produzir o desenvolvimento de práticas em saúde de acordo com os pressupostos da promoção da saúde. De modo, a superar o modelo biomédico, ainda presente de forma camuflada nas políticas e programas voltados para a saúde do adolescente, caracterizadas por uma assistência preventivista, impossibilitando a implementação de práticas promotoras de saúde. 


\section{Considerações Finais}

O estudo ao buscar conhecer a percepção de adolescentes acerca da relação saúde e meio ambiente, evidenciou que esta relação é relacionada a temas como poluição, desmatamento, mudanças climáticas como fatores que interferem no processo saúdedoença, observando a conceituação de saúde como ausência de saúde. Destaca a necessidade de práticas de educação em saúde com temas transversais e pluralista, para que essa população possa reconhecer o meio ambiente como um espaço vivo, que os danos causados, sofridos pode materializar uma relação de causa e efeito para o processo saúde-doença. Assim, há necessidade de mudanças nas práticas em saúde com os adolescentes para que estes se empoderem e sejam capazes de realizarem práticas sustentáveis e promotoras de saúde.

\section{Referências}

Alves, S. A. A., Moreira, D. A. A., Callou, R. S. B.L. et al. (2019). Estratégias de ensino aprendizagem na graduação de enfermagem: uma reflexão à luz do referencial teórico de Paulo Freire, International Journal of Development Research, 09,(10), 30938-30941.

Alves, S. A. A., Oliveira, M. L. B. (2018). Sociocultural aspects of health and disease and their pragmatic impact. J Hum Growth Dev. 28(2):183-188. doi: http://dx.doi.org/10.7322/jhgd.147236

Anhas, D. M., Castro-Silva, C. R. (2017). Sentidos atribuídos por adolescentes e jovens à saúde: desafios da Saúde da Família em uma comunidade vulnerável de Cubatão, São Paulo, Brasil. Saúde Soc. São Paulo, v.26, n.2, p.484-495. doi: https://doi.org/ 10.1590/S0104-12902017169735

Araújo, M. M. L., Pequeno, A. M. C., Guimarães, J. M. X., Vasconcelos Filho., J. O., Carneiro, C., \& Negreiros, F. D. S. (2018). Processo de ensino-aprendizagem de enfermagem: reflexões de docentes sobre o estágio curricular supervisionado. Investigação Qualitativa em Saúde//Investigación Cualitativa en Salud//Volume 2. Atas CIAIQ.

Bardin, L. (2009). Análise de Conteúdo. Lisboa, Portugal; Edições 70, LDA.

Baggio, M. A., Berres, R., Gregolin, B. P. S., Aikes, S. (2018). Introduction of the School Health Program in the city of Cascavel, Parana State: report of nurses. Rev Bras Enferm [Internet]. 71(Suppl 4):1540-7. [Thematic Issue: Education and teaching in Nursing] doi: http://dx.doi.org/10.1590/0034-7167-2017-0188

Bezerra, A. C. V. (2017). Vigilância em saúde ambiental no Brasil: heranças e desafios. Saúde Soc. São Paulo, v. 28, n. 4, p. 1044-1057.

Brasil, E. G. M., Silva, R. M., Silva, M. R. F., Rodrigues, D. P., \& Queiroz, M.V. O. (2017). Adolescent health promotion and the School Health Program: complexity in the articulation of health and education. Rev Esc Enferm USP. 51 :e03276. doi: http://dx.doi.org/10.1590/S1980220X2016039303276

Brasil. (2016). Decreto $n^{\circ} 8.892$, de 27 de outubro de 2016. Diário Oficial da República Federativa do Brasil, Brasília (DF), out 31; Seção 1.

Brasil. (2013). Ministério da Saúde. Secretaria de Atenção à Saúde. Departamento de Ações Programáticas Estratégicas. Orientações básicas de atenção integral à saúde de adolescentes nas escolas e unidades básicas de saúde. Brasília: Editora do Ministério da Saúde.

Busato, M. A., Ferraz, L., Frank, N. L. P. (2015). Reflexões sobre a relação saúde e ambiente: a percepção de uma comunidade. HOLOS, ano 31, Vol. 6. doi: 10.15628holos.

Buss, P. M., Chamas, C., Miriam Faid, M., Morel, C. (2016). Desenvolvimento, saúde e política internacional: a dimensão da pesquisa \& inovação. Cad. Saúde Pública, Rio de Janeiro, 32 Sup 2: e00046815. doi: https://doi.org/10.1590/0103-311X00046815 
Buss, P. M. (2000). Promoção da saúde e qualidade de vida. Ciência \& Saúde Coletiva, Rio de Janeiro, v. 5, n. 1, p. 163-177.

Chiesa, A. M., Nascimento, D. D. G., Braccial, L. A. D., Oliveira, M. A. C., Ciampone, M. \& H. T. (2007). A formação de profissionais da saúde: Aprendizagem significativa à luz da promoção da saúde. Cogitare Enferm, 12: p.236-40.

Dias, G. L., Camponogara, S., Costa, V. Z., Cezar-Vaz, M. R., Weiller, T. H., \& Cardoso, L. S. (2018). Representações sociais sobre saúde e meio ambiente para equipes de Estratégia Saúde da Família. Saúde Soc. São Paulo, v.27, n.1, p. 163-174.

Djonú, P., Rabelo, L. S., Lima, P. V. P. S., Souto, M. V. S., Sabadia, J. A. B., \& Sucupira, P. R. G. J. (2018). Objetivos do desenvolvimento sustentável e condições de saúde em áreas de risco. Ambient. soc. vol.21. São Paulo. Epub 29-Nov.

Farre, A. G. M. C., Pinheiro, P. N. C., Vieira, N. F. C., Gubert, F. A., Alves, M. D. S., \& Monteiro, E. M. L. M. (2018). Adolescent health promotion based on community-centered arts education. Rev Bras Enferm [Internet]. 71(1):26-33. doi: http://dx.doi.org/10.1590/0034-7167-20

FairalL L, Bateman E. (2017). Health workers are vital to sustainable development goals and universal health coverage. BMJ, v. 356, j1357.

Fernandes, E. S. F., Santos, A.M. (2020). Desencontros entre formação profissional e necessidades de cuidado aos adolescentes na Atenção Básica à Saúde. Interface (Botucatu). 24: e190049 doi: https://doi.org/10.1590/Interface.190049

Farre A. G. M. C., Pinheiro P. N. C., Vieira N. F. C., Gubert F. A., Alves M. D. S., \& Monteiro E. M L. M. (2018). Adolescent health promotion based on community-centered arts education. Rev Bras Enferm [Internet]. 71(1):26-33. doi: http://dx.doi.org/10.1590/0034-7167-20

Freire, P. (2011). Pedagogia do Oprimido. Rio de Janeiro: Paz e Terra.

Guha, I., Maliye, C. H., Gupta, S. S., Garg, B. S. (2019). Qualitative Assessment of Life Skill Development of Adolescent Girls through Kishori Panchayat: An Adolescents for Health Action Model in Selected Villages of Rural Central India. Indian J Community Med. Jul-Set; 44 (3): 265-270. doi: 10.4103 / ijcm.IJCM_74_19.

Jing, S. et al. (2016). Vulnerability assessment of urban ecosystems driven by water resources, human health and atmospheric environment. Journal of Hydrology, Amsterdam, v. 536, p. 457-470.

Kruk Margaret, E., Gage, Anna D., Joseph Naima, T., Danaei, Goodarz., García-Saisó, Sebastián., \& Salomon Joshua, A. (2018). Mortality due to low-quality health systems in the universal health coverage era: a systematic analysis of amenable deaths in 137 countries. Lancet. 392 2203-12. doi: doi.org/10.1016/ S0140-6736(18)31668-4

Lopes, I. E., Nogueira, J. A. D., Rocha, D. G. (2018). Eixos de ação do Programa Saúde na Escola e Promoção da Saúde: revisão integrativa. Saúde Debate, V. 42, N. 118, P. 773-789, JULSET. doi: 10.1590/0103-1104201811819

Lee, Y., Kim, S. Y. (2019). Public health law coverage in support of the health-related sustainable development goals (SDGs) among 33 Western Pacific countries. Globalization And Health [Global Health], ISSN: 1744-8603. Apr 11; Vol. 15 (1), pp. 29; Publisher: BioMed Central; PMID: 30971269

Machado, M. F. A. S., Gubert, F. A., Meye, A. P. G. F. V., Sampaio, Y. P. C. C., Dias, M. A. S., Almeida, A. M. B., Morais, A. P. P., Silva, A. C., Campos, J. S., Chagas, M. I. O., \& Chaves, E. S. C. (2015). Programa saúde na escola: estratégia promotora de saúde na atenção básica no brasil. Journal of Human Growth and Development. 25(3): 307-312.

Monteiro, I. F. C., Monteiro, P. D. E. B. S. C. O. (2017). A educação ambiental e as representações sociais dos professores da rede pública no ensino fundamental. RevBEA. 12(1):165-76. doi: 10.34024/revbea.2017.v12.2391

Machado, J. M. H., Martins, W. J., Souza, M. S; Fenner, A. L. D., Missifany Silveira, M., \& Machado, A. A. (2017). Territórios saudáveis e sustentáveis: contribuição para saúde coletiva, desenvolvimento sustentável e governança territorial. Com. Ciências Saúde. 28(2):243 249. 
Oliveira, F. P. S. L., Vargas, A. M. D., Hartz, C., Dias, S., Fereira, E. F. (2018). Percepção de escolares do ensino fundamental sobre o Programa Saúde na Escola: um estudo de caso em Belo Horizonte, Brasil. Ciências \& Saúde. 23 (9): 2891- 2898. doi: 10.1590/1413-81232018239.16582018

Porto, M. F., Finamore, R. (2012). Riscos, saúde e justiça ambiental: o protagonismo das populações atingidas na produção de conhecimento. Ciênc. saúde coletiva [online]. vol.17, n.6, pp.1493-1501. ISSN 1413 8123. doi: http://dx.doi.org/10.1590/S141381232012000600013.

Pereira, C. V., Alves, S. A. A., Sobreira, C. L. S., Lopes, M. S. V. (2021). Educação ambiental e arboviroses no contexto escolar. Rev enferm UFPE on line. 15:e244683. 2021. doi: https://doi.org/10.5205/1981-8963.2021.244683

Rangel, V. et al. (2015). Considerações para uma agenda estratégica de saúde e ambiente e sustentabilidade: horizontes da Fiocruz para 2022. In: BRASIL. Ministério da Saúde. Saúde e ambiente para as populações do campo, da floresta e das águas. Brasília, DF. p. 47-61

Santos, S. B., Machado, A. P. A., Sampaio, L. A., Abreu, L. C., \& Bezerra, I. M. P. (2019). Acquired Syphilis: construction and validation of educational technology for adolescents. J Hum Growth Dev 29(1): 65-74. doi: http://dx.doi.org/10.7322/jhgd.157752

Schaefer, R., Barbiani, R., Nora, C. R. D., Viegas, K., Leal, S. M. C., Lora, O. S., Ciconet, R., \& Micheletti, V. D. (2018). Políticas de Saúde de adolescentes e jovens no contexto lusobrasileiro: especificidades e aproximações. Ciênc. saúde colet. 23 (9) Set. doi: https://doi.org/10.1590/1413-81232018239.11202018

Silva, J. P., Gonçalves, M. F. C., Andrade, L. S., Monteiro, E. M. L. M., \& Silva, M. A. I. (2018). Promoção da saúde na educação básica: percepções dos alunos de licenciatura em enfermagem. Rev Gaúcha Enferm. 39:e2017-0237.

Tong, A., Sainsbury, P., Craig, J. (2007). Critérios consolidados para relatar a pesquisa qualitativa (COREQ): uma lista de verificação de 32 itens para entrevistas e grupos focais. Revista Internacional de Qualidade em Saúde 19 (6), 349357. doi: Http://dx.doi.org/10.1093/intghc/mzm042.

Usa. (2011). United Nations Children's Fund. The state of the world's children 2011: adolescence an age of opportunity[Internet]. New York (NY): UNICEF [cited 2013 Jun 20]. Available from: https://www.unicef.org/sowc2011/pdfs/SOWC-2011-Main- Report_EN_02092011.pdf

Ventura, D. F. L., Ribeiro, H., DI Guilio, G. M., Jaime, P. C., Nunes, J., Bógus, C. M., Antunes, J. L. F., \& Waldman, E. A. (2020). Challenges of the COVID-19 pandemic: for a Brazilian research agenda in global health and sustainability. Cad. Saúde Pública; 36(4):e00040620. doi: 10.1590/0102-311X00040620 